\title{
Quantitative three-dimensional cardiovascular magnetic resonance myocardial perfusion imaging in systole and diastole
}

Manish Motwani ${ }^{1}$, Ananth Kidambi ${ }^{1}$, Steven Sourbron², Timothy A Fairbairn ${ }^{1}$, Akhlaque Uddin' ${ }^{1}$ Sebastian Kozerke ${ }^{3}$, John P Greenwood ${ }^{1}$ and Sven Plein ${ }^{1 *}$

\begin{abstract}
Background: Two-dimensional (2D) perfusion cardiovascular magnetic resonance (CMR) remains limited by a lack of complete myocardial coverage. Three-dimensional (3D) perfusion CMR addresses this limitation and has recently been shown to be clinically feasible. However, the feasibility and potential clinical utility of quantitative 3D perfusion measurements, as already shown with 2D-perfusion CMR and positron emission tomography, has yet to be evaluated. The influence of systolic or diastolic acquisition on myocardial blood flow (MBF) estimates, diagnostic accuracy and image quality is also unknown for 3D-perfusion CMR. The purpose of this study was to establish the feasibility of quantitative 3D-perfusion CMR for the detection of coronary artery disease (CAD) and to compare systolic and diastolic estimates of MBF.
\end{abstract}

Methods: Thirty-five patients underwent 3D-perfusion CMR with data acquired at both end-systole and mid-diastole. MBF and myocardial perfusion reserve (MPR) were estimated on a per patient and per territory basis by Fermi-constrained deconvolution. Significant CAD was defined as stenosis $\geq 70 \%$ on quantitative coronary angiography.

Results: Twenty patients had significant CAD (involving 38 out of 105 territories). Stress MBF and MPR had a high diagnostic accuracy for the detection of CAD in both systole (area under curve [AUC]: 0.95 and 0.92, respectively) and diastole (AUC: 0.95 and 0.94). There were no significant differences in the AUCs between systole and diastole ( $p$ values $>0.05$ ). At stress, diastolic MBF estimates were significantly greater than systolic estimates (no CAD: $3.21 \pm 0.50$ vs. $2.75 \pm 0.42 \mathrm{ml} / \mathrm{g} / \mathrm{min}, p<0.0001 ;$ CAD: $2.13 \pm 0.45$ vs. $1.98 \pm 0.41 \mathrm{ml} / \mathrm{g} / \mathrm{min}, \mathrm{p}<0.0001$ ); but at rest, there were no significant differences ( $p$ values $>0.05$ ). Image quality was higher in systole than diastole (median score 3 vs. 2 , $p=0.002)$.

Conclusions: Quantitative 3D-perfusion CMR is feasible. Estimates of MBF are significantly different for systole and diastole at stress but diagnostic accuracy to detect CAD is high for both cardiac phases. Better image quality suggests that systolic data acquisition may be preferable.

Keywords: Cardiovascular magnetic resonance, Perfusion, 3-dimensional, Myocardial perfusion imaging, Ischemic heart disease, Myocardial blood flow

\footnotetext{
* Correspondence: s.plein@leeds.ac.uk

'Multidisciplinary Cardiovascular Research Centre \& The Division of

Cardiovascular and Diabetes Research, Leeds Institute of Genetics, Health \&

Therapeutics, University of Leeds, Leeds, UK

Full list of author information is available at the end of the article
} 


\section{Background}

Myocardial perfusion imaging with cardiovascular magnetic resonance (CMR) is a highly accurate technique for the detection of coronary artery disease (CAD) [1]. However, conventional acquisition with two-dimensional (2D) methods can only acquire a small number of noncontiguous slices of the left ventricle (LV) at each R-R interval, and therefore incomplete myocardial coverage remains a significant limitation.

Recent technological advances have allowed unprecedented acceleration of dynamic CMR and have led to the development of three-dimensional (3D) myocardial perfusion CMR methods providing full LV coverage with preserved temporal and spatial resolution [2-4]. Three recent studies have shown $3 \mathrm{D}$-perfusion CMR to be clinically feasible and highly accurate for the detection of CAD with visual perfusion assessment [5-7]. However, the feasibility and potential clinical application of deriving quantitative estimates of myocardial blood flow (MBF) from 3D-perfusion CMR has not yet been studied.

A further limitation of conventional 2D-perfusion CMR is that each slice is acquired in a different period of the cardiac cycle. Two recent quantitative studies have shown a significant difference in MBF estimates derived from the same mid-ventricular slice acquired in systole and diastole with 2D-perfusion CMR $[8,9]$. As well as limiting quantitative comparisons between slices, these significant phasic differences impact on inter-study and longitudinal comparisons of MBF. Unlike 2D-perfusion CMR, 3D imaging allows acquisition of data from the entire myocardium in the same, optimised period of the cardiac cycle. Most previous 3D-perfusion CMR studies have acquired data in systole but to date it is unknown whether systolic or diastolic acquisition leads to better image quality and diagnostic yield. Furthermore, it is unknown whether quantitative estimates of MBF from 3D data demonstrate the same phasic differences previously reported for $2 \mathrm{D}$ techniques $[8,9]$.

The purpose of this study was therefore to establish the feasibility of quantitative 3D-perfusion CMR for the detection of coronary artery disease (CAD) and to compare systolic and diastolic estimates of MBF. Defining the optimal cardiac phase for acquisition may be more relevant for $3 \mathrm{D}$ than $2 \mathrm{D}$-perfusion CMR because it allows the acquisition of all slices in a particular cardiac phase.

\section{Methods}

\section{Population}

Forty consecutive patients with known or suspected CAD were recruited. All patients were imaged within 30 days of clinically scheduled diagnostic coronary angiography. No revascularization or clinical events occurred between angiography and CMR. Exclusion criteria were contraindications to CMR, adenosine, or gadolinium contrast agents, recent myocardial infarction (MI) or unstable angina (within 6 months), or poorly controlled arrhythmias. Patients were instructed to refrain from caffeine for 24 hours before their CMR study but continue cardiac medications as normal. The study was approved by the regional ethics committee and all patients gave written consent.

\section{CMR protocol}

All studies were performed on a $3.0 \mathrm{~T}$ scanner (Achieva TX, Philips Healthcare, Best, The Netherlands) equipped with dual-source parallel radiofrequency transmission technology and a 32-channel cardiac coil. For perfusion imaging, a 3D spoiled turbo gradient-echo sequence was used (TR/TE/flip angle $1.8 \mathrm{~ms} / 0.7 \mathrm{~ms} / 15^{\circ}$; saturation prepulse delay $150 \mathrm{~ms}$; linear $k$-space encoding; 70\% partial Fourier acquisition in two dimensions; typical field of view $350 \times$ $350 \mathrm{~mm} ; 10$ fold $k-t$ acceleration and 11 training profiles leading to a net acceleration of 7.0; typical acquisition duration $192 \mathrm{~ms}, k-t$ principal component analysis (PCA) reconstruction; reconstructed to 12 contiguous slices with voxel size $2.3 \times 2.3 \times 5 \mathrm{~mm}^{3}$ ) [2,7].

Two $k-t$ undersampled $3 \mathrm{D}$ data sets were acquired in each R-R interval, each preceded by a non-selective saturation prepulse. Vertical and horizontal long-axis cine images were used to identify appropriate trigger delays for systolic and diastolic acquisition [8-10]. Additionally, because of the longitudinal lengthening of the heart from systole to diastole, the position of the end-systolic and mid-diastolic perfusion stacks (12 slices each) were individually planned from the chosen systolic and diastolic cine frames (Figure 1) $[8,9]$. The same trigger delays were used for stress and rest acquisitions.

Stress perfusion images were acquired during intravenous adenosine-induced hyperemia administered for $4 \mathrm{~min}$ at $140 \mathrm{mcg} / \mathrm{kg} / \mathrm{min}$. Consistent with previous 3D-perfusion CMR studies, an intravenous bolus of $0.075 \mathrm{mmol} / \mathrm{kg}$ of gadobutrol (Gadovist, Bayer Schering Pharma, Berlin, Germany) was administered at a rate of $4.0 \mathrm{ml} / \mathrm{s}$ followed by a $20 \mathrm{ml}$ saline flush. Stress perfusion CMR was followed by cine imaging covering the left ventricle in 10-12 shortaxis sections. Rest perfusion CMR was performed 15 min after stress, using identical imaging parameters. Late gadolinium enhancement (LGE) imaging was acquired in the same short-axis geometry as perfusion imaging after an additional 10-15 min using conventional 2D methods (T1 weighted segmented inversion recovery gradient echo; TR/ TE/flip angle $4.9 \mathrm{~ms} / 1.9 \mathrm{~ms} / 15^{\circ}$; inversion time individually adjusted according to Look-Locker scan; spatial resolution $1.35 \times 1.35 \times 10 \mathrm{~mm}$ ).

\section{Image quality}

Systolic and diastolic perfusion images were analyzed in separate reporting sessions in random order (MM, 2 years 

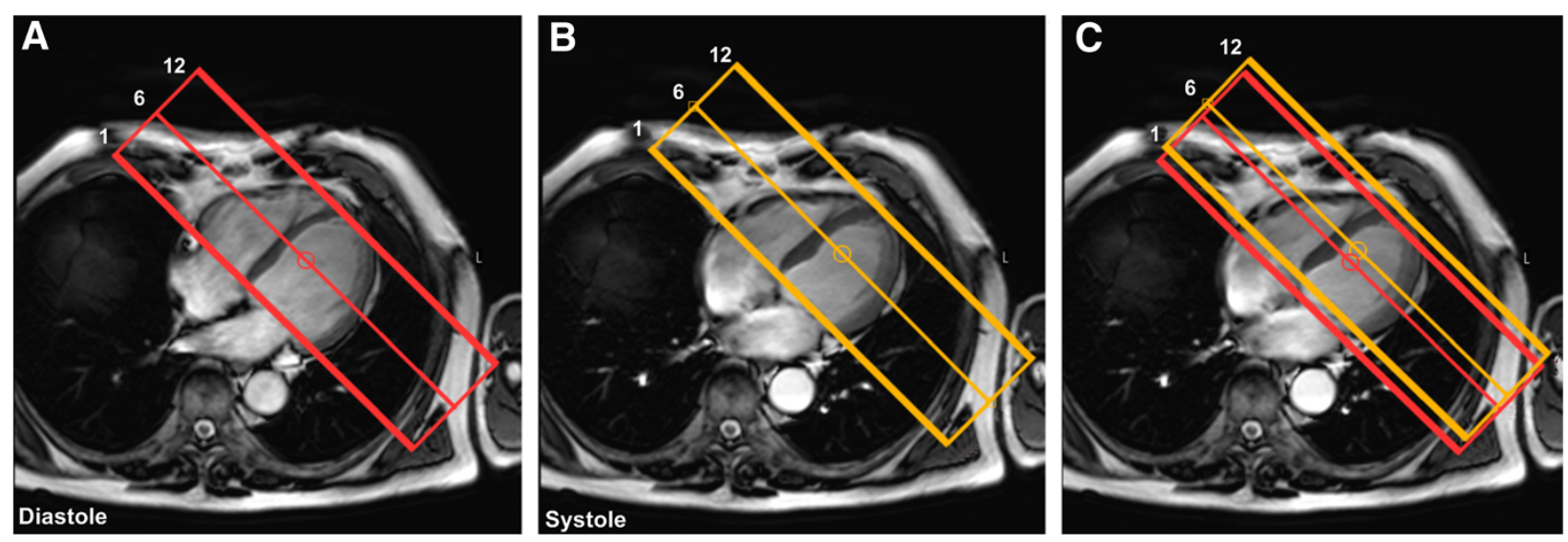

Figure 1 Acquisition planning. Because of the longitudinal lengthening of the heart from systole to diastole, the position of the mid-diastolic (red) and end-systolic perfusion stacks (yellow) (12 slices each) were individually planned from the chosen diastolic (Panel A) and systolic

(Panel B) 4-chamber cine frames. Panel $\mathbf{C}$ shows both stacks superimposed on the chosen end-systolic frame.

experience of perfusion CMR). Overall image quality was scored as follows: 0 =non-diagnostic, 1 = poor, $2=$ adequate and $3=$ excellent. The occurrence of artifact related to respiratory-motion, $k-t$ reconstruction or darkrim artifact was scored as follows: $0=$ none, $1=$ mild, $2=$ moderate and $3=$ severe.

\section{Myocardial blood flow estimation}

Perfusion images were processed offline using previously validated in-house software (PMI 0.4; written in IDL 6.4 (ITT Visual Information Systems, Boulder, CO) [11]. All short-axis slices with clearly identifiable LV cavity enhancement during first-pass perfusion and with $>75 \%$ circumferential LV myocardium were included in the analysis $[5,6]$.

\section{Per patient analysis}

Following manual rigid motion-correction, a circular region of interest (ROI) was drawn in the basal LV cavity in diastole, to derive the arterial input function (AIF). The same (diastolic) AIF was used for both systolic and diastolic estimates of MBF in order to avoid potential variations in the AIF between phases with subsequent effects on MBF estimation [8].

A whole-heart myocardial region of interest (ROI) excluding any dark-rim artifact was drawn for both systolic and diastolic perfusion images (covering all slices containing myocardium). Signal intensity-time data were converted to concentration-time data by subtracting the baseline signal, and global MBF was estimated at stress and rest using constrained deconvolution with a delayed Fermi-model applied to the first pass $[9,12,13]$. MPR was calculated as stress MBF divided by rest MBF.

\section{Per territory analysis}

The above analysis was repeated on a per territory basis using the 17-segment AHA model adjusted for coronary dominance [14]. For this, all slices from an individual patient were first visually allocated to basal, mid or apical sections of the model. For each perfusion territory, a myocardial ROI was then outlined including all segments pertaining to that territory across all slices according to the 17-segment AHA model. MBF and MPR estimates were obtained using the same algorithm as for the whole-heart ROI.

\section{Intra-observer and inter-observer variability}

Thirty random territories were re-analyzed 1 month later by the same observer (M.M.) and by a second observer A.K. ( 2 yrs and 1 yr experience respectively). A.K. was blinded to the results of all previous analyses.

\section{Quantitative coronary angiography}

Quantitative coronary angiography was performed (QCAPlus, Sanders Data Systems, Palo Alto, CA, USA) on anonymised X-ray angiography images (M.M. 6 years experience in coronary angiography). Significant CAD was defined as luminal stenosis $\geq 70 \%$ diameter in any of the main epicardial coronary arteries or their branches with a diameter of $\geq 2 \mathrm{~mm}$.

\section{Statistical analysis}

Analysis was performed using SPSS 17.0 (SPSS, Chicago, IL). Data are presented as mean \pm SD. Group means were compared using paired or unpaired Student t-tests; or within-subjects analysis of variance with GreenhouseGeisser correction for multi-sample sphericity, as appropriate. Ordinal data were compared using the Wilcoxon signed-rank test. Receiver-operating characteristic (ROC) analysis was performed on a per territory basis, to determine the diagnostic accuracy of MPR to detect significant CAD. Diagnostic accuracies are presented as area under the ROC curve (AUC); and were compared between 
systole and diastole using methods described by Delong and Delong. Optimal MPR cut-off values, for both cardiac phases, were defined as values that maximised the sum of sensitivity and specificity. A secondary ROC analysis was performed to evaluate the diagnostic accuracy of stress MBF diastolic/systolic ratio. To assess reproducibility, the coefficients of variation $(\mathrm{CoV})$ for intra- and inter-observer measurements were calculated. Because three coronary territories were examined per patient, the intra-cluster correlation coefficient (ICC) was calculated for MPR estimates to determine the design effect and the need to adjust data for clustering. All statistical tests were 2tailed and a p value $<0.05$ was considered significant.

\section{Results}

\section{Study population}

Five of the 40 recruited patients were excluded: 3 because of claustrophobia and 2 owing to technical problems (1 mistimed contrast injection; 1 significantly altered patient position between stress and rest scans). A total of 35 patients (105 coronary territories) were therefore available for analysis. Table 1 shows the baseline patient characteristics. QCA confirmed significant CAD in 20 patients (57\%) and 38 coronary territories (36\%). Only 3 patients had evidence of MI on LGE imaging (the same 3

Table 1 Patient characteristics

\begin{tabular}{|c|c|}
\hline Parameter & Data $(n=35)$ \\
\hline Age (yrs \pm SD) & $62 \pm 8$ \\
\hline \multicolumn{2}{|l|}{ Sex, n (\%) } \\
\hline Male & $26(74)$ \\
\hline Female & $9(26)$ \\
\hline \multicolumn{2}{|l|}{ Risk factors, n (\%) } \\
\hline Hypertension & $18(51)$ \\
\hline Hypercholesterolemia & $19(54)$ \\
\hline Diabetes Mellitus & $6(17)$ \\
\hline Smoking & $14(40)$ \\
\hline Previous MI & $3(9)$ \\
\hline Previous $\mathrm{PCl}$ & $3(9)$ \\
\hline \multicolumn{2}{|c|}{ Angiography findings, $\mathrm{n}(\%)$ * } \\
\hline No significant disease & $15(43)$ \\
\hline One-vessel disease & $6(17)$ \\
\hline Two-vessel disease & $10(29)$ \\
\hline Three -vessel disease & $4(11)$ \\
\hline LAD disease & $17(49)$ \\
\hline LCX disease & $10(29)$ \\
\hline RCA disease & $11(31)$ \\
\hline
\end{tabular}

*Significant disease defined as coronary stenosis $\geq 70 \%$ on quantitative coronary analysis.

$M I=$ myocardial infarction; $P C I=$ percutaneous coronary intervention; $L A D=$ left anterior descending coronary artery; $L C X=$ left circumflex coronary artery; $R C A=$ right coronary artery. patients with a clinical history of $\mathrm{MI})$, and this involved only 3 of the 105 territories analysed. Figure 2 shows an example of the stress perfusion images acquired in a patient with significant CAD.

\section{Image quality}

Overall image quality was better in systole than in diastole (median image quality score: 3 vs. 2 respectively; $\mathrm{p}=0.002)$. In diastole, there was a greater frequency of dark-rim artifact (19 patients [54\%] vs. 9 patients [26\%] and a higher overall artifact score compared to systole (median scores: 1 vs. 0 respectively; $\mathrm{p}<0.0001$ ).

In 5 patients $(14 \%)$, perfusion images (both cardiac phases) were affected by $k-t$ reconstruction artifacts at stress and/or rest due to respiratory motion, but all of these artifacts occurred at the end of the breath-hold and did not affect analysis of the first-pass perfusion images.

\section{Myocardial blood flow estimation}

Estimates of MBF and MPR for both cardiac phases are seen in Tables 2, 3 and 4 . On per patient $(n=35)$ and per territory analysis $(n=105)$, mean resting MBF was similar in both cardiac phases (all $\mathrm{p}$ values $>0.05$ ); but mean stress MBF and MPR were significantly greater in diastole than systole (all p values <0.001). These relationships existed in normal and CAD subgroups, as well as overall (all p values <0.01) (Tables 2, 3, 4). In both cardiac phases, stress MBF and MPR were significantly lower in the presence of CAD than in normal patients (all p values $<0.01$ ) or normal territories (all p values < 0.0001) (Tables 2, 3, 4).

Analysis of the normal patient group $(n=15)$ found no significant regional differences in stress $\mathrm{MBF}$, rest $\mathrm{MBF}$ or MPR between the LAD, LCX or RCA perfusion territories in both phases (all p values <0.05) (Table 5).

Based on MPR estimates, the ICC for this study was low ( $r 1=0.09$ [95\% CI: -0.25 to 0.41$]$ ) with a design effect of 1.18 where cluster size $=3$. This shows that the study design of using 3 'related' coronary territories per patient $(\mathrm{n}=35)$ to derive a sample size of 105 coronary territories does not significantly diminish statistical power.

\section{Diagnostic performance Stress MBF}

On a per territory analysis $(n=105)$, the use of stress MBF alone had a high overall diagnostic accuracy for the detection of CAD - which was similar in both cardiac phases $(\mathrm{AUC}=0.95$ for both; $\mathrm{p}=0.70)$. The optimal stress $\mathrm{MBF}$ cut-off value was $2.31 \mathrm{ml} / \mathrm{min} / \mathrm{g}$ for systole and $2.60 \mathrm{ml} / \mathrm{min} / \mathrm{g}$ for diastole. At these thresholds, the sensitivity and specificity were $92 \%$ and $93 \%$ respectively for systole; and $95 \%$ and $96 \%$ for diastole. There was no significant difference between the diagnostic accuracy of 


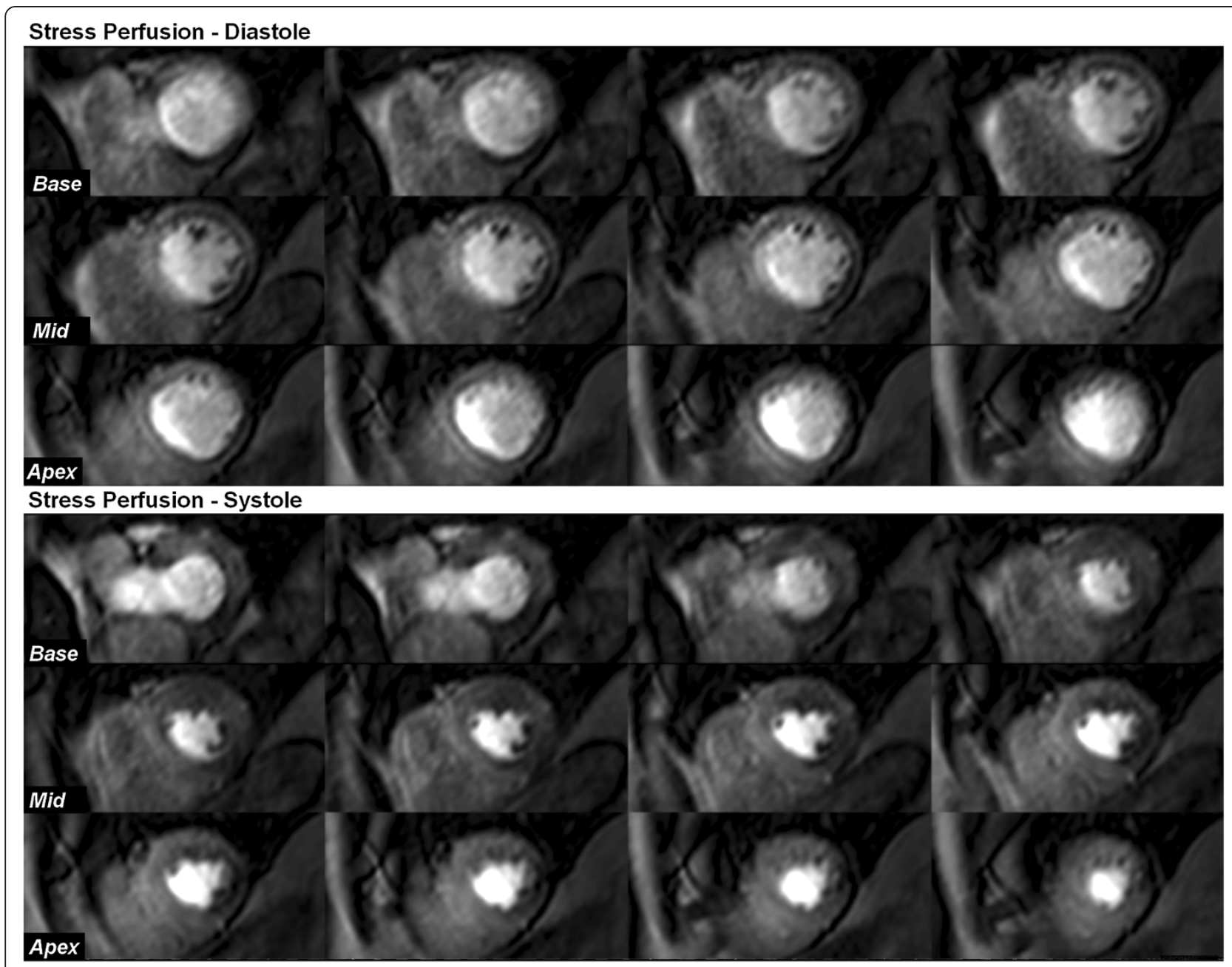

\section{LGE Imaging}

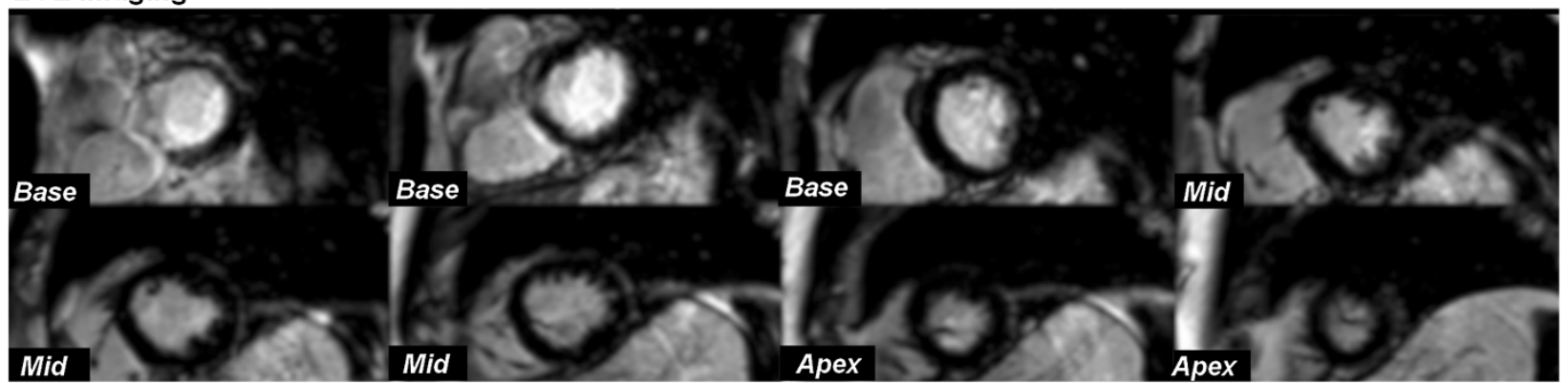

Figure 2 Case example: 3D-perfusion CMR in systole and diastole. This example shows 3D-perfusion CMR in a 75-year-old man with angina. Stress-induced perfusion defects are seen infero-laterally from base to apex and antero-laterally from mid-ventricle to apex in both diastole and systole. However, perfusion defects are difficult to discern from dark-rim artifact in diastole and are more clearly delineated with systolic acquisition. Late-gadolinium enhancement imaging did not reveal any myocardial infarction. X-ray coronary angiography revealed $80 \%$ stenosis of a large diagonal branch and significant proximal disease in a large dominant left circumflex artery.

MPR or stress MBF alone for either cardiac phase (both $\mathrm{p}$ values $>0.05$ ) (Figure 3).

\section{MPR}

On per territory analysis $(\mathrm{n}=105)$, MPR also had a high overall diagnostic accuracy for the detection of significant
CAD, and this was similar in both cardiac phases (AUC, systole: 0.92 vs. diastole: $0.94 ; \mathrm{p}=0.41$ ) (Figure 3). The optimal MPR cut-off value was 1.75 for systole and 2.02 for diastole (Figure 4). At these thresholds, the sensitivity and specificity were $82 \%$ and $93 \%$ respectively for systole; and $87 \%$ and $94 \%$ for diastole. The diagnostic accuracy of 
Table 2 Estimates of MBF and MPR from 3D perfusion CMR - per patient analysis

\begin{tabular}{|c|c|c|c|c|c|c|c|c|c|}
\hline & \multicolumn{3}{|c|}{ Stress MBF $(\mathrm{ml} / \mathrm{min} / \mathrm{g})$} & \multicolumn{3}{|c|}{ Rest MBF $(\mathrm{ml} / \mathrm{min} / \mathrm{g})$} & \multicolumn{3}{|c|}{ MPR } \\
\hline & Systole & Diastole & $p$ & Systole & Diastole & $p$ & Systole & Diastole & $p$ \\
\hline Normal $(n=15)$ & $2.88 \pm 0.32$ & $3.47 \pm 0.41$ & $p<0.0001$ & $1.28 \pm 0.17$ & $1.26 \pm 0.15$ & $p=0.45$ & $2.27 \pm 0.37$ & $2.78 \pm 0.40$ & $p<0.0001$ \\
\hline$C A D(n=20)$ & $2.32 \pm 0.42$ & $2.53 \pm 0.47$ & $p<0.0001$ & $1.32 \pm 0.19$ & $1.28 \pm 0.21$ & $p=0.06$ & $1.82 \pm 0.54$ & $2.08 \pm 0.74$ & $p<0.001$ \\
\hline Overall $(n=35)$ & $2.56 \pm 0.47$ & $2.93 \pm 0.65$ & $p<0.0001$ & $1.30 \pm 0.18$ & $1.27 \pm 0.19$ & $p=0.06$ & $2.01 \pm 0.08$ & $2.38 \pm 0.05$ & $p<0.0001$ \\
\hline
\end{tabular}

All values expressed as mean \pm SD. Coronary artery disease $(C A D)$ defined as stenosis $\geq 70 \%$. $C M R=$ cardiovascular magnetic resonance; $M B F=$ myocardial blood flow; $M P R=$ myocardial perfusion reserve.

MPR to detect CAD in each of the 3 coronary territories is shown in Table 6 and no significant differences were seen between cardiac phases.

\section{Diastolic/systolic stress MBF ratio}

The diastolic/systolic stress MBF ratio was significantly lower for territories with CAD than in normal territories $(1.07 \pm 0.06$ vs. $1.17 \pm 0.11 ; \mathrm{p}<0.0001)$. On ROC analysis, the diagnostic accuracy (AUC) of this ratio to detect significant CAD was 0.79 . The optimal cut-off value for the ratio was 1.10 which gave a sensitivity of $82 \%$ and specificity of $76 \%$ (Figure 5).

\section{Reproducibility}

\section{Stress MBF}

The mean absolute difference between intra-observer measurements of stress MBF was similar in systole and diastole $(0.33 \pm 0.14$ vs. $0.35 \pm 0.16 ; \mathrm{p}=0.18)$; and the corresponding CoVs were $16 \%$ and $17 \%$ respectively. The mean absolute difference between inter-observer measurements of stress MBF was also similar in systole and diastole $(0.41 \pm$ 0.22 vs. $0.45 \pm 0.20 ; \mathrm{p}=0.11$ ) with corresponding CoVs of $18 \%$ for both.

\section{MPR}

The mean absolute difference between intra-observer measurements of MPR was similar in systole and diastole $(0.30 \pm 0.15$ vs. $0.36 \pm 0.13 ; \mathrm{p}=0.09)$; and the corresponding CoVs were both $18 \%$. The mean absolute difference between inter-observer measurements of MPR was also similar in systole and diastole $(0.35 \pm 0.17$ vs. $0.41 \pm 0.15$; $\mathrm{p}=0.07$ ) with corresponding CoVs of $20 \%$ and $21 \%$ respectively.

\section{Discussion}

The main findings of this study are 1) quantitative 3Dperfusion CMR is feasible and has a high diagnostic accuracy for the detection of CAD; 2) similar to 2D studies, estimates of stress MBF and MPR from 3D data are significantly greater in diastole than systole; and 3) the diastolic dominance of stress MBF estimates is reduced in ischemia.

One of the limitations of myocardial perfusion imaging and standard visual interpretation is the dependence on a reference area of normal perfusion. This is a particular impediment in diffuse or balanced multi-vessel disease. This limitation can be avoided by using absolute quantification of MBF [15]. At present, the most robust technique to quantify MBF noninvasively is positron emission tomography (PET) - but its wide-spread clinical application has been slowed by limited access [16]. PET imaging also involves exposure to ionizing radiation, and its spatial resolution limits evaluation of transmural flow differences in normal thickness myocardium.

Over the last decade, several animal, normal volunteer and patient studies have validated the use of CMR for absolute MBF quantification against microsphere and invasive coronary flow reserve measurements [17-19]. Furthermore, several clinical studies have demonstrated high diagnostic accuracy of CMR derived estimates of absolute MBF and MPR against both QCA and fractional flow reserve $[9,13,15,20,21]$. Nonetheless, the lack of complete myocardial coverage has been a significant limitation of conventional 2D-perfusion CMR for this purpose.

This study has for the first time demonstrated the feasibility of quantitative whole-heart 3D-perfusion CMR. Shin et al have previously reported semi-quantitative measures (time-intensity curve indices) of resting

Table 3 Estimates of MBF and MPR from 3D-perfusion CMR - per territory analysis

\begin{tabular}{|c|c|c|c|c|c|c|c|c|c|}
\hline & \multicolumn{3}{|c|}{ Stress MBF $(\mathrm{ml} / \mathrm{min} / \mathrm{g})$} & \multicolumn{3}{|c|}{ Rest MBF $(\mathrm{ml} / \mathrm{min} / \mathrm{g})$} & \multicolumn{3}{|c|}{ MPR } \\
\hline & Systole & Diastole & $p$ & Systole & Diastole & $p$ & Systole & Diastole & $p$ \\
\hline Normal $(n=67)$ & $2.75 \pm 0.42$ & $3.21 \pm 0.50$ & $p<0.0001$ & $1.24 \pm 0.15$ & $1.25 \pm 0.15$ & $p=0.27$ & $2.26 \pm 0.43$ & $2.59 \pm 0.44$ & $p<0.0001$ \\
\hline$C A D(n=38)$ & $1.98 \pm 0.41$ & $2.13 \pm 0.55$ & $p<0.0001$ & $1.24 \pm 0.15$ & $1.26 \pm 0.14$ & $p=0.20$ & $1.63 \pm 0.14$ & $1.72 \pm 0.19$ & $p<0.01$ \\
\hline Overall $(n=105)$ & $2.47 \pm 0.55$ & $2.82 \pm 0.71$ & $p<0.0001$ & $1.24 \pm 0.15$ & $1.25 \pm 0.15$ & $p=0.10$ & $2.03 \pm 0.52$ & $2.27 \pm 0.61$ & $p<0.0001$ \\
\hline
\end{tabular}

All values expressed as mean \pm SD. Coronary artery disease $(C A D)$ defined as stenosis $\geq 70 \%$. $C M R=$ cardiovascular magnetic resonance; $M B F=$ myocardial blood flow; $M P R=$ myocardial perfusion reserve. 
Table 4 Regional estimates of MBF and MPR - per territory $(n=105)$

\begin{tabular}{|c|c|c|c|c|c|c|}
\hline & \multicolumn{3}{|c|}{ Normal } & \multicolumn{3}{|c|}{ CAD } \\
\hline & Systole & Diastole & $p$ & Systole & Diastole & $p$ \\
\hline \multicolumn{7}{|c|}{ Stress MBF $(\mathrm{ml} / \mathrm{min} / \mathrm{g})$} \\
\hline$\angle A D$ & $2.91 \pm 0.35$ & $3.43 \pm 0.46$ & $p<0.0001$ & $2.11 \pm 0.46$ & $2.28 \pm 0.52$ & $p<0.001$ \\
\hline$L C X$ & $2.63 \pm 0.33$ & $3.10 \pm 0.44$ & $p<0.0001$ & $1.90 \pm 0.28$ & $2.04 \pm 0.28$ & $p<0.01$ \\
\hline$R C A$ & $2.77 \pm 0.51$ & $3.16 \pm 0.55$ & $p<0.0001$ & $1.85 \pm 0.39$ & $1.97 \pm 0.40$ & $p<0.0001$ \\
\hline \multicolumn{7}{|c|}{ Rest MBF (ml/min/g) } \\
\hline$\angle A D$ & $1.26 \pm 0.17$ & $1.30 \pm 0.17$ & $p=0.10$ & $1.21 \pm 0.12$ & $1.23 \pm 0.12$ & $p=0.52$ \\
\hline$L C X$ & $1.26 \pm 0.17$ & $1.24 \pm 0.13$ & $p=0.56$ & $1.22 \pm 0.14$ & $1.20 \pm 0.10$ & $p=0.27$ \\
\hline$R C A$ & $1.20 \pm 0.13$ & $1.23 \pm 0.15$ & $p=0.28$ & $1.28 \pm 0.13$ & $1.24 \pm 0.16$ & $p=0.26$ \\
\hline \multicolumn{7}{|l|}{ MPR } \\
\hline$\angle A D$ & $2.35 \pm 0.42$ & $2.68 \pm 0.48$ & $p<0.0001$ & $1.75 \pm 0.37$ & $1.87 \pm 0.51$ & $p=0.02$ \\
\hline$L C X$ & $2.12 \pm 0.35$ & $2.52 \pm 0.38$ & $p<0.0001$ & $1.58 \pm 0.32$ & $1.72 \pm 0.29$ & $p<0.01$ \\
\hline$R C A$ & $2.33 \pm 0.51$ & $2.59 \pm 0.48$ & $p<0.001$ & $1.46 \pm 0.34$ & $1.60 \pm 0.34$ & $p<0.01$ \\
\hline
\end{tabular}

All values expressed as mean \pm SD. Coronary artery disease $(C A D)$ defined as stenosis $\geq 70 \%$. $M B F=$ myocardial blood flow; $M P R=$ myocardial perfusion reserve; $L A D=$ left anterior descending coronary artery; $L C X=$ left circumflex coronary artery; $R C A=$ right coronary artery.

myocardial perfusion from a 3D acquisition in 3 healthy volunteers - but no stress acquisition or absolute MBF quantification was performed [10]. The MBF values derived with $3 \mathrm{D}$-perfusion $\mathrm{CMR}$ in normal patients in the present study are comparable to values from PET studies and the previous CMR literature [22,23]. For example, in a large study of 160 healthy men and women with PET, the mean resting $\mathrm{MBF}$ was $0.98 \pm 0.23 \mathrm{ml} / \mathrm{min} / \mathrm{g}$ (range 0.59 $2.05 \mathrm{ml} / \mathrm{min} / \mathrm{g}$ ) and the mean stress $\mathrm{MBF}$ was $3.77 \pm$ $0.85 \mathrm{ml} / \mathrm{min} / \mathrm{g}$ (range $1.85-5.99 \mathrm{ml} / \mathrm{min} / \mathrm{g}$ ) [23]. Intra- and inter-observer reproducibility for stress MBF and MPR in our study was also similar to that seen in 2D-perfusion CMR and PET studies [24,25].

The finding of lower estimates of stress MBF in systole compared to diastole is consistent with the expected physiology and a number of previous studies. Physiologically,

Table 5 Comparison of regional MBF and MPR estimates in normal patients $(n=15)$

\begin{tabular}{|c|c|c|c|c|}
\hline & \multicolumn{4}{|c|}{ Perfusion territory } \\
\hline & $L A D$ & $L C X$ & $R C A$ & $p$ \\
\hline \multicolumn{5}{|c|}{ Stress MBF (ml/min/g) } \\
\hline Systole & $2.95 \pm 0.36$ & $2.66 \pm 0.29$ & $2.88 \pm 0.46$ & $P=0.11$ \\
\hline Diastole & $3.51 \pm 0.46$ & $3.19 \pm 0.27$ & $3.27 \pm 0.47$ & $P=0.10$ \\
\hline \multicolumn{5}{|c|}{ Rest MBF (ml/min/g) } \\
\hline Systole & $1.27 \pm 0.17$ & $1.29 \pm 0.19$ & $1.20 \pm 0.13$ & $p=0.25$ \\
\hline Diastole & $1.29 \pm 0.17$ & $1.27 \pm 0.14$ & $1.22 \pm 0.15$ & $p=0.47$ \\
\hline \multicolumn{5}{|l|}{ MPR } \\
\hline Systole & $2.36 \pm 0.42$ & $2.10 \pm 0.32$ & $2.45 \pm 0.53$ & $P=0.06$ \\
\hline Diastole & $2.76 \pm 0.45$ & $2.54 \pm 0.33$ & $2.71 \pm 0.48$ & $P=0.27$ \\
\hline
\end{tabular}

All values expressed as mean $\pm \mathrm{SD} . M B F=$ myocardial blood flow; $M P R=$ myocardial perfusion reserve; $L A D=$ left anterior descending coronary artery; $L C X=$ left circumflex coronary artery; $R C A=$ right coronary artery. one explanation is that during systole, the effect of adenosine-mediated vasodilatation is diminished by the compression of intramyocardial vessels [26]. Two previous 2D-perfusion CMR studies have shown the same phasic differences with higher stress MBF estimates in diastole, but no difference between the phases at rest $[8,9]$. One previous 3D-perfusion CMR study confirmed similar semi-quantitative measures of resting myocardial perfusion between systole and diastole - but no stress perfusion was performed [10]. Our study has now demonstrated that these phasic differences are also seen with 3Dperfusion CMR quantification and underline the importance of stating the phase of acquisition in future studies to allow comparison in the literature.

Quantitative analysis with MPR yielded high diagnostic accuracies in both systole and diastole (AUC: 0.92 and 0.94 respectively). The optimal MPR cut-off values for detecting significant CAD (1.75 for systole and 2.02 for diastole) were within the range of 1.50-2.06 reported in previous 2D-perfusion CMR studies [9,13,20,21,27]. Recently, in 2D-perfusion CMR, Huber et al $(\mathrm{n}=31)$ showed that the use of stress $\mathrm{MBF}$ alone had a similar diagnostic accuracy as MPR (AUC 0.92 vs. 0.84 respectively; $\mathrm{p}<0.18$ ) [11]. Our study has shown a similar finding in 3Dperfusion CMR and the implication is that a rest perfusion sequence could potentially be omitted in quantitative studies, thus reducing both scanning and post-processing times without a loss in diagnostic yield (Figure 3).

The noted reduction in diastolic/systolic stress MBF ratio in territories with CAD is consistent with previous invasive studies measuring coronary flow velocity throughout the cardiac cycle [28]. The loss of diastolic dominance has been explained by the increased influence of a significant stenosis on flow during periods of low vascular resistance 

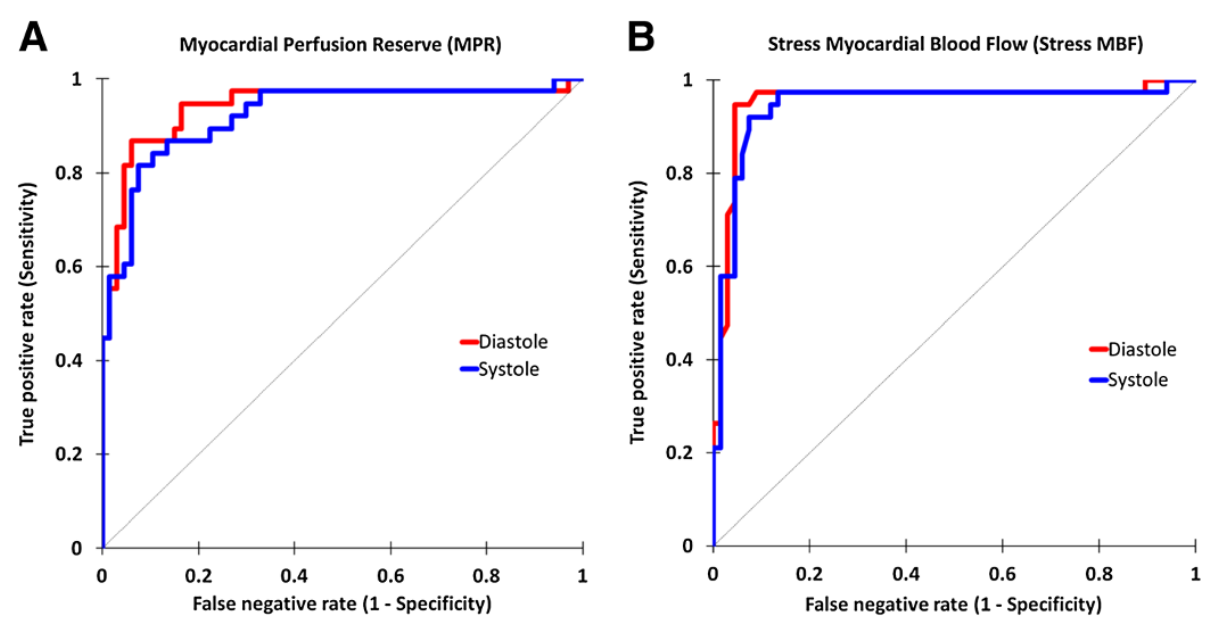

Figure 3 Diagnostic accuracy of quantitative 3D-perfusion CMR. (A) Receiver-operator characteristic curves shows a high diagnostic accuracy in both systole and diastole for MPR (area under curve [AUC]: 0.92 vs. 0.94 respectively; $p=0.41$ ). (B) Use of stress MBF alone also had a high diagnostic accuracy in both cardiac phases (AUC: 0.95 for both; $p=0.70$ ) and in fact there was no significant difference compared to MPR ( $p>0.05$ for both cardiac phases).

and low intramyocardial tension (diastole); as compared with that during periods of high vascular resistance and high intramyocardial tension (systole) [29]. As such, the diastolic/systolic stress MBF ratio is a novel diagnostic index with moderate diagnostic accuracy $(\mathrm{AUC}=0.79)-$ and this may warrant further evaluation in future studies.

In our quantitative study, both phases had similar diagnostic performance and reproducibility. However, similar to previous 2D studies, diastole was more prone to dark-rim artifact with an adverse effect on image quality; and this is thought to relate to the thinner myocardium, making it more prone to partial volume effects at a given spatial resolution [9]. For this reason, as well as the fact that contour delineation is easier in systole because of the thicker myocardium, we would suggest systole as the preferred phase for 3D-perfusion CMR acquisition - particularly for quantitative studies. Although analysis time was not specifically measured, each $3 \mathrm{D}$ perfusion dataset took approximately $20 \mathrm{~min}$ to analyse on a per patient basis (including stress and rest analyses for either the systolic or diastolic cardiac phase). Quantifying 3D-perfusion CMR can be simpler than quantifying conventional 2D datasets, because as in our study, fewer dynamic images are often acquired and there is a degree

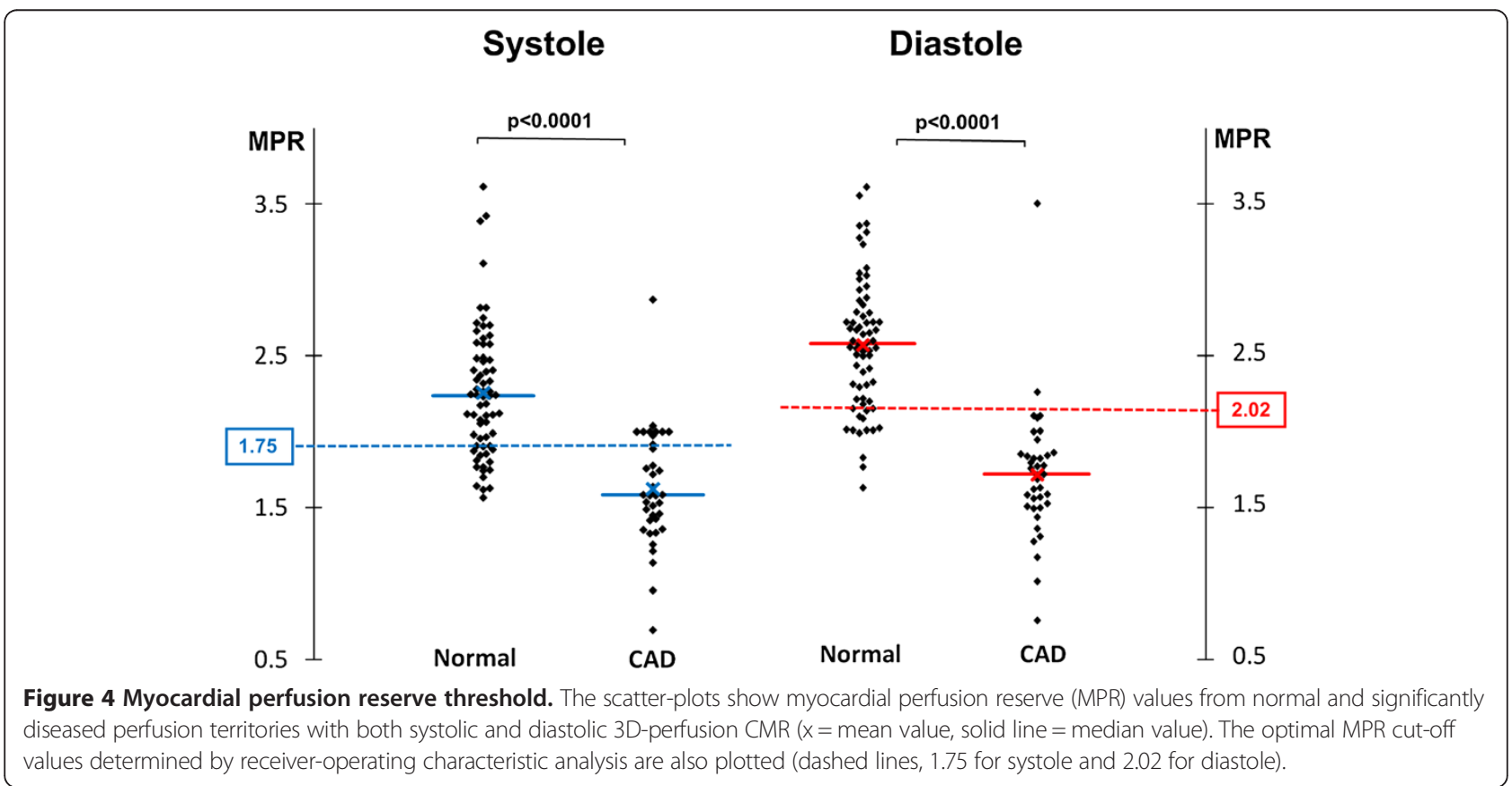


Table 6 Diagnostic accuracy of quantitative 3D-perfusion CMR-per territory $(n=105)$

\begin{tabular}{lccc}
\hline & \multicolumn{3}{c}{ AUC for MPR } \\
\cline { 2 - 4 } & Systole & Diastole & $\boldsymbol{P}$ \\
\hline All territories & $0.92(0.87-0.98)$ & $0.94(0.88-0.99)$ & $\mathrm{p}=0.41$ \\
LAD & $0.89(0.78-0.99)$ & $0.90(0.79-1.00)$ & $\mathrm{p}=0.76$ \\
LCX & $0.88(0.77-0.99)$ & $0.98(0.93-1.00)$ & $\mathrm{p}=0.34$ \\
RCA & $0.92(0.86-0.99)$ & $0.98(0.93-1.00)$ & $\mathrm{p}=0.50$ \\
\hline
\end{tabular}

Values are area under the curve $(A \cup C)$ and $(95 \%$ confidence interval). $L A D=$ left anterior descending coronary artery; $L C X=$ left circumflex coronary artery; $R C A=$ right coronary artery

of temporal filtering due to the undersampling in the temporal domain which reduces the amount of time-consuming manual motion correction required.

Finally, there is considerable scope for quantitative perfusion CMR in clinical practice and therefore demonstrating the feasibility of $3 \mathrm{D}$ whole-heart coverage and quantification is important. Nonetheless, there still remain a number of other limitations that hold back the wider clinical adoption of quantitative perfusion CMR. The current lack of standardisation in image acquisition, contrast dosing protocols, post-processing, mathematical modelling and interpretation is addressed by an international standardisation task force [30,31]. There is also no widely available and validated analysis software for quantitative analysis of perfusion CMR data and research groups generally use in-house solutions. Analysis can be time-consuming, precluding routine clinical application. Finally, the incremental value of quantitative analysis of myocardial perfusion CMR analysis needs to be shown in large clinical studies.

\section{Study limitations}

The spatio-temporal undersampling methods required for $3 \mathrm{D}$ data acquisition are sensitive to respiratory motion, cardiac arrhythmia and low-pass temporal filtering - all of which pose additional challenges to quantitative assessment. Low-pass temporal filtering in particular may lead to underestimation of MBF. We reduced these limitations by use of the constrained $k-t$ PCA framework for image reconstruction, which has been shown to improve temporal fidelity, permitting robust measurements of MBF at very high acceleration factors [32]. The latter is also less prone to respiratory artifact as temporal basis functions are derived based on the low-resolution training data acquired in every heartbeat [2].

Although MPR performed well in our study, perfusion imaging is a measurement of the hemodynamic consequences of a stenosis rather than its anatomy, and therefore our use of QCA is an imperfect reference standard. Following this initial feasibility study, future validation against fractional flow reserve is planned in a larger clinical population. Another limitation, common to many previous studies, is the potential effect of data clustering as three perfusion territories are examined per patient $[20,27]$. However, the design effect of this was low (1.18) owing to a small ICC and cluster size.

Finally, the model used for estimating MBF assumes a linear relation between signal and contrast agent concentration i.e. ignoring saturation effects in the LV blood pool which can lead to underestimation of MBF [33]. This is particularly relevant as we used a relatively high contrast agent dose of $0.075 \mathrm{mmol} / \mathrm{kg}$ bodyweight to be consistent with previous 3D-perfusion CMR studies. Proposed solutions include the use of a non-linear signal model combined with precontrast T1-mapping and/or the use of a small pre-bolus to measure the AIF. There is currently no evidence that either of these potential solutions actually leads to improved diagnostic accuracy for the detection of CAD in the clinical setting. In fact
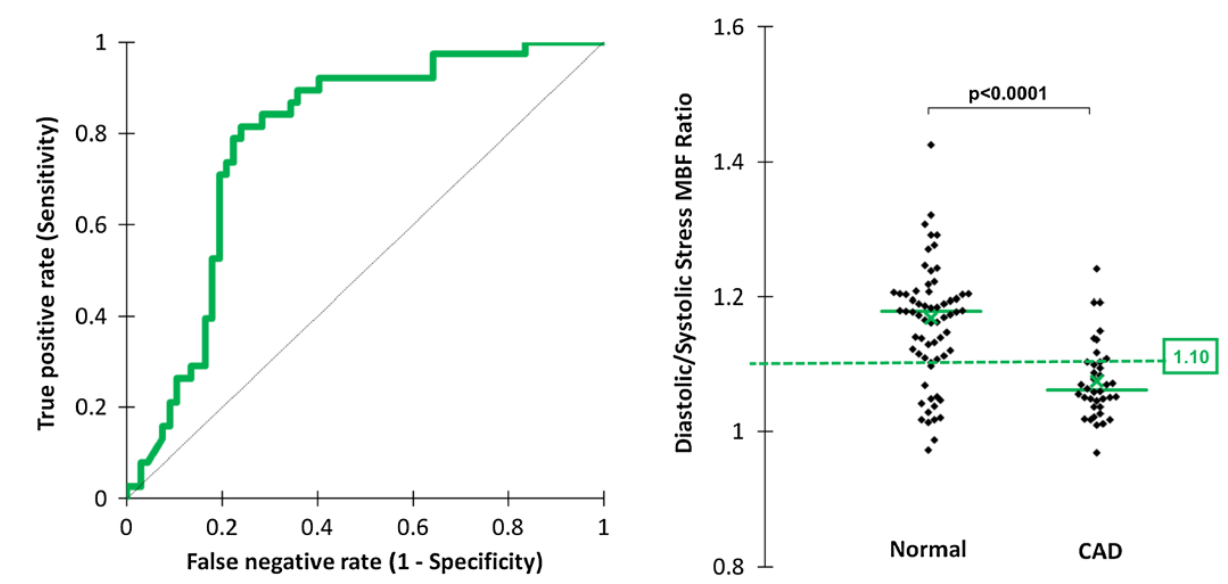

Figure 5 Diastolic/systolic stress myocardial blood flow ratio. The ratio of diastolic to systolic myocardial blood flow at stress was significantly lower for territories with coronary artery disease (CAD) than in normal territories $(1.07 \pm 0.06$ vs. $1.17 \pm 0.11 ; p<0.0001)$. On receiver-operator characteristic analysis, the diagnostic accuracy (area under the curve) of this ratio to detect significant CAD was 0.79 . The optimal cut-off value for the ratio was 1.10, which gave a sensitivity of $82 \%$ and specificity of $76 \%$. 
the only study directly addressing this question came to the opposite conclusion i.e. the use of a pre-bolus AIF was found to reduce diagnostic accuracy compared to a single-bolus approach [34].

\section{Conclusions}

We have shown that quantitative 3D-perfusion CMR is feasible and can be used to detect CAD with high diagnostic accuracy. In addition, we have found that there are significant differences in systolic and diastolic MBF estimates. Both cardiac phases provide comparable diagnostic yield, albeit at different thresholds. Because systolic images had fewer artifacts and higher image quality, systole may be the preferred phase for acquisition of 3D perfusion data.

\section{Abbreviations}

CAD: Coronary artery disease; CMR: Cardiovascular magnetic resonance; MBF: Myocardial blood flow; MPR: Myocardial perfusion reserve; $k$-t: $k$-space and time; PCA: Principal component analysis; ROI: Region of interest; AIF: Arterial input function; AUC: Area-under-the-curve; ROC: Receiver-operator characteristic.

\section{Competing interests}

The authors declare that they have no competing interests. SP and AK are funded by a British Heart Foundation Senior Clinical Research Fellowship (FS/1062/28409). SP and JPG receive an educational research grant from Philips Healthcare.

\section{Authors' contributions}

MM: conception and design, acquisition, analysis and interpretation of data, drafting of manuscript; AK: acquisition, analysis and interpretation of data, critical and intellectual revision of manuscript; SS: analysis and interpretation of data, critical and intellectual revision of manuscript; TAF: critical and intellectual revision of manuscript; AU: acquisition of data, critical and intellectual revision of manuscript; SK: critical and intellectual revision of manuscript; JPG: interpretation of data, critical and intellectual revision of manuscript; SP: conception and design, interpretation of data, critical and intellectual revision of manuscript. All authors read and approved the final manuscript.

\section{Acknowledgements}

We thank Gavin Bainbridge, Margaret Saysell and Caroline Richmond (radiographers) for their technical assistance; and Petra Bijsterveld and Fiona Richards (research nurses) for their assistance with patient recruitment.

\section{Author details}

'Multidisciplinary Cardiovascular Research Centre \& The Division of Cardiovascular and Diabetes Research, Leeds Institute of Genetics, Health \& Therapeutics, University of Leeds, Leeds, UK. ${ }^{2}$ Division of Medical Physics, University of Leeds, Leeds, UK. ${ }^{3}$ Institute for Biomedical Engineering, University and ETH Zurich, Zurich, Switzerland.

Received: 9 October 2013 Accepted: 29 January 2014 Published: 24 February 2014

\section{References}

1. Greenwood JP, Maredia N, Younger JF, Brown JM, Nixon J, Everett CC, Bijsterveld P, Ridgway JP, Radjenovic A, Dickinson CJ, Ball SG, Plein S. Cardiovascular magnetic resonance and single-photon emission computed tomography for diagnosis of coronary heart disease (CE-MARC): a prospective trial. Lancet. 2012; 379:453-460.

2. Vitanis V, Manka R, Giese D, Pedersen H, Plein S, Boesiger P, Kozerke S. High resolution three-dimensional cardiac perfusion imaging using compartment-based k-t principal component analysis. Magn Reson Med. 2011; 65:575-587.
3. Shin T, Nayak KS, Santos JM, Nishimura DG, Hu BS, McConnell MV. Three-dimensional first-pass myocardial perfusion MRI using a stack-of-spirals acquisition. Magn Reson Med. 2013; 69:839-844.

4. Chen L, Adluru G, Schabel MC, McGann CJ, Dibella EV. Myocardial perfusion MRI with an undersampled 3D stack-of-stars sequence. Med Phys. 2012; 39:5204-5211.

5. Manka R, Jahnke C, Kozerke S, Vitanis V, Crelier G, Gebker R, Schnackenburg B, Boesiger P, Fleck E, Paetsch I. Dynamic 3-dimensional stress cardiac magnetic resonance perfusion imaging: detection of coronary artery disease and volumetry of myocardial hypoenhancement before and after coronary stenting. J Am Coll Cardiol. 2011; 57:437-444.

6. Manka R, Paetsch I, Kozerke S, Moccetti M, Hoffmann R, Schroeder J, Reith S, Schnackenburg B, Gaemperli O, Wissmann L, Wyss CA, Kaufmann PA, Corti R, Boesiger P, Marx N, Lüscher TF, Jahnke C. Whole-heart dynamic three-dimensional magnetic resonance perfusion imaging for the detection of coronary artery disease defined by fractional flow reserve: determination of volumetric myocardial ischaemic burden and coronary lesion location. Eur Heart J. 2012; 33:2016-2024.

7. Jogiya R, Kozerke S, Morton G, De Silva K, Redwood S, Perera D, Nagel E, Plein S. Validation of dynamic 3-dimensional whole heart magnetic resonance myocardial perfusion imaging against fractional flow reserve for the detection of significant coronary artery disease. J Am Coll Cardiol. 2012; 60:756-765.

8. Radjenovic A, Biglands JD, Larghat A, Ridgway JP, Ball SG, Greenwood JP, Jerosch-Herold M, Plein S. Estimates of systolic and diastolic myocardial blood flow by dynamic contrast-enhanced MRI. Magn Reson Med. 2010; 1703:1696-1703.

9. Motwani M, Fairbairn TA, Larghat A, Mather AN, Biglands JD. Systolic Versus Diastolic Acquisition in Myocardial Perfusion MR Imaging. Radiology. 2012; 262:816-23.

10. Shin T, Pohost GM, Nayak KS. Systolic 3D first-pass myocardial perfusion MRI: Comparison with diastolic imaging in healthy subjects. Magn Reson Med. 2010; 63:858-64.

11. Huber A, Sourbron S, Klauss V, Schaefer J, Bauner KU, Schweyer M, Reiser M, Rummeny E, Rieber J. Magnetic resonance perfusion of the myocardium: semiquantitative and quantitative evaluation in comparison with coronary angiography and fractional flow reserve. Invest Radiol. 2012; 47:332-338.

12. Jerosch-Herold M, Wilke N, Stillman AE, Wilson RF. Magnetic resonance quantification of the myocardial perfusion reserve with a Fermi function model for constrained deconvolution. Med Phys. 1998; 25:73-84.

13. Costa MA, Shoemaker S, Futamatsu H, Klassen C, Angiolillo DJ, Nguyen M, Siuciak A, Gilmore P, Zenni MM, Guzman L, Bass TA, Wilke N. Quantitative magnetic resonance perfusion imaging detects anatomic and physiologic coronary artery disease as measured by coronary angiography and fractional flow reserve. J Am Coll Cardiol. 2007; 50:514-522.

14. Cerqueira MD, Weissman NJ, Dilsizian V, Jacobs AK, Kaul S, Laskey WK, Pennell DJ, Rumberger JA, Ryan T, Verani MS. Standardized myocardial segmentation and nomenclature for tomographic imaging of the heart: a statement for healthcare professionals from the Cardiac Imaging Committee of the Council on Clinical Cardiology of the American Heart Association. Circulation. 2002; 105:539-542.

15. Patel AR, Antkowiak PF, Nandalur KR, West AM, Salerno M, Arora V, Christopher J, Epstein FH, Kramer CM. Assessment of Advanced Coronary Artery Disease Advantages of Quantitative Cardiac Magnetic Resonance Perfusion Analysis. J Am Coll Cardiol. 2010; 56:561-569.

16. Kajander SA, Joutsiniemi E, Saraste M, Pietilä M, Ukkonen H, Saraste A, Sipilä HT, Teräs M, Mäki M, Airaksinen J. Clinical Value of Absolute Quantification of Myocardial Perfusion With 150-Water in Coronary Artery Disease Clinical Perspective. Circ Cardiovasc Imaging. 2011; 4:678-684.

17. Christian TF, Rettmann DW, Aletras AH, Liao SL, Taylor JL, Balaban RS, Arai AE. Absolute Myocardial Perfusion in Canines Measured by Using Dual-Bolus First-Pass MR Imaging. Radiology. 2004; 232:677-684.

18. Jerosch-Herold M, Swingen C, Seethamraju RT. Myocardial blood flow quantification with MRI by model-independent deconvolution. Med Phys. 2002; 29:886-897.

19. Wilke $N$, Jerosch-Herold M, Wang $Y$, Huang $Y$, Christensen BV, Stillman AE, Ugurbil K, McDonald K, Wilson RF. Myocardial perfusion reserve: assessment with multisection, quantitative, first-pass MR imaging Radiology. 1997; 204:373-384. 
20. Lockie T, Ishida M, Perera D, Chiribiri A, De Silva K, Kozerke S, Marber M, Nagel E, Rezavi R, Redwood S, Plein S. High-resolution magnetic resonance myocardial perfusion imaging at 3.0-Tesla to detect hemodynamically significant coronary stenoses as determined by fractional flow reserve. J Am Coll Cardiol. 2011; 57:70-75.

21. Futamatsu H, Wilke N, Klassen C, Shoemaker S, Angiolillo DJ, Siuciak A, Morikawa-Futamatsu K, Suzuki N, von Ziegler F, Bass TA, Costa MA. Evaluation of cardiac magnetic resonance imaging parameters to detect anatomically and hemodynamically significant coronary artery disease. Am Heart J. 2007; 154:298-305.

22. Sdringola S, Johnson NP, Kirkeeide RL, Cid E, Gould KL. Impact of Unexpected Factors on Quantitative Myocardial Perfusion and Coronary Flow Reserve in Young, Asymptomatic Volunteers. J Am Coll Cardiol Img. 2011; 4:402-412.

23. Chareonthaitawee P, Kaufmann PA, Rimoldi O, Camici PG. Heterogeneity of resting and hyperemic myocardial blood flow in healthy humans. Cardiovasc Res. 2001; 50:151-161.

24. Morton G, Chiribiri A, Ishida M, Hussain ST, Schuster A, Indermuehle A, Perera D, Knuuti J, Baker S, Hedström E, Schleyer P, O'Doherty M, Barrington S, Nagel E. Quantification of absolute myocardial perfusion in patients with coronary artery disease: comparison between cardiovascular magnetic resonance and positron emission tomography. J Am Coll Cardiol. 2012; 60:1546-1555.

25. Larghat AM, Maredia N, Biglands J, Greenwood JP, Ball SG, Jerosch-Herold M, Radjenovic A, Plein S. Reproducibility of first-pass cardiovascular magnetic resonance myocardial perfusion. J Magn Reson Imaging. 2013; 874:865-874.

26. Hiramatsu O, Goto M, Yada T, Kimura A, Chiba Y, Tachibana H, Ogasawara Y, Tsujioka K, Kajiya F. In vivo observations of the intramural arterioles and venules in beating canine hearts. J Physiol. 1998; 509:619-628.

27. Rieber J, Huber A, Erhard I, Mueller S, Schweyer M, Koenig A, Schiele TM, Theisen K, Siebert U, Schoenberg SO, Reiser M, Klauss V. Cardiac magnetic resonance perfusion imaging for the functional assessment of coronary artery disease: a comparison with coronary angiography and fractional flow reserve. Eur Heart J. 2006; 27:1465-1471.

28. Segal J, Kern MJ, Scott NA, King SB, Doucette JW, Heuser RR, Ofili E, Siegel R. Alterations of phasic coronary artery flow velocity humans during percutaneous coronary angioplasty. J Am Coll Cardiol. 1992; 20:276-286.

29. Spaan JA, Breuls NP, Laird JD. Diastolic-systolic coronary flow differences are caused by intramyocardial pump action in the anesthetized dog. Circ Res. 1981; 49:584-593.

30. Kramer CM, Barkhausen J, Flamm SD, Kim RJ, Nagel E. Standardized cardiovascular magnetic resonance (CMR) protocols 2013 update. J Cardiovasc Magn Reson. 2013; 15:91.

31. Schulz-Menger J, Bluemke DA, Bremerich J, Flamm SD, Fogel MA, Friedrich MG, Kim RJ, von Knobelsdorff-Brenkenhoff F, Kramer CM, Pennell DJ, Plein S, Nagel E. Standardized image interpretation and post processing in cardiovascular magnetic resonance: Society for Cardiovascular Magnetic Resonance (SCMR) Board of Trustees Task Force on Standardized Post Processing. J Cardiovasc Magn Reson. 2013; 15:35.

32. Pedersen H, Kozerke S, Ringgaard S, Nehrke K, Kim WY. k-t PCA: temporally constrained k-t BLAST reconstruction using principal component analysis. Magn Reson Med. 2009; 62:706-716.

33. Sourbron S. Technical aspects of MR perfusion. Eur J Radiol. 2010; 76:304-313.

34. Groothuis JG, Kremers FP, Beek AM, Brinckman SL, Tuinenburg AC, Jerosch-Herold M, van Rossum AC, Hofman MB. Comparison of dual to single contrast bolus magnetic resonance myocardial perfusion imaging for detection of significant coronary artery disease. J Magn Reson Imaging. 2010; 32:88-93.

doi:10.1186/1532-429X-16-19

Cite this article as: Motwani et al.: Quantitative three-dimensional cardiovascular magnetic resonance myocardial perfusion imaging in systole and diastole. Journal of Cardiovascular Magnetic Resonance 2014 16:19.

\section{Submit your next manuscript to BioMed Central and take full advantage of:}

- Convenient online submission

- Thorough peer review

- No space constraints or color figure charges

- Immediate publication on acceptance

- Inclusion in PubMed, CAS, Scopus and Google Scholar

- Research which is freely available for redistribution

Submit your manuscript at www.biomedcentral.com/submit
C Biomed Central 